\title{
Effects of Phenyl Hydrogen Polysiloxane Molecular Structure on the Performance of LED Packaging Silicone Rubber
}

\author{
Huaijun $\mathrm{ZHU}^{1,2 *}$, Zilin DAI ${ }^{1}$, Weiping $\mathrm{TU}^{2}$ \\ ${ }^{1}$ Guangdong Research Institute of Rare Metals, Guangdong Academy of Sciences, Guangzhou 510651, China \\ ${ }^{2}$ School of Chemistry and Chemical Engineering, South China University of Technology, Guangzhou 510640, China \\ crossref http://dx.doi.org/10.5755/j01.ms.24.2.18494
}

Received 29 June 2017; accepted 23 August 2017

\begin{abstract}
Phenyl hydrogen polysiloxanes of different structures were prepared, and LED packaging silicone rubbers were made from these polysiloxanes. The hardness, elongation, tensile strength and anti-yellowing of the LED packaging silicone rubbers on lamps were characterized. The results show that phenyl hydrogen polysiloxanes of resin structures had lower molecular weight, and that the cured silicone rubbers exhibited higher hardness, tensile strength, crosslink density and lower gas permeability. Phenyl hydrogen polysiloxanes of oil structures had higher molecular weight, and the cured silicone rubbers from these polysiloxanes exhibited lower volume expansion coefficient, higher hot and cold impact cycles and less change in hardness during the aging process. The study results further showed that the degree of change in hardness, yellowing, gas permeability, sulfuration and volume expansion coefficient was reduced by using phenyl hydrogen polysiloxanes of resin-oil structures as crosslinking agents, enabling the combined performance advantages of both resin-structure and oil-structure rubbers.

Keywords: polysiloxane, sulfuration, LED, packaging silicone rubber, yellowing.
\end{abstract}

\section{INTRODUCTION}

Phenyl silicone rubbers are organic silicon products with special performance properties such as high and low temperature resistance, ultraviolet resistance, radiation resistance, anti-aging, and high refractive index [1-4]. Due to their excellent properties, these silicone rubbers have become more reliable LED encapsulation materials than epoxy resins $[5,6]$.

LED packaging silicone rubbers can prevent the chips from oxidation and sulfuration in the air, improve the light emission efficiency and brightness of emitting diode, and extend the service lives of the chips. LED encapsulated silicone rubbers are usually prepared by the hydrosilylation reaction of vinyl-containing polysiloxane and hydrogencontaining polysiloxane under the catalysis of platinum compound. Good mechanical properties, optical properties, hot and cold impact resistance, heat resistance of the silicone rubbers are required.

LED encapsulated silicone rubbers can be divided into methyl silicone rubbers and phenyl silicone rubbers. Phenyl hydrogen polysiloxane as a cross-linking agent plays a decisive role in the performance of phenyl silicone rubber.

Phenyl hydrogen polysiloxanes of different structures impart different mechanical and optical properties to the rubbers prepared from them [7,8]. Studying the effects of phenyl hydrogen polysiloxane structures on the performance of phenyl silicone rubbers has important significance for the design, synthesis and application of LED packaging silicone rubbers $[9,10]$.

Phenyl hydrogen polysiloxanes can be classified into two basic structural types: oil-structure (linear structure)

\footnotetext{
* Corresponding author. Tel.: +086-020-37238801; fax: +086-020-

37238405. E-mail address: zhuhuaijun@163.com (H. Zhu)
}

and resin-structure (network structure). In this study, phenyl hydrogen polysiloxanes of different structures were prepared, and LED packaging silicone rubbers were made from these polysiloxanes. The hardness, elongation, tensile strength and anti-yellowing of the LED packaging silicone rubbers on lamps were characterized, in an attempt to resolve the problems (e.g. change in hardness, yellowing, gas permeation and sulfuration, and high volume expansion coefficient) encountered in application of silicone rubbers in LED packaging.

\section{EXPERIMENTAL DETAILS}

\subsection{Chemicals and reagents}

Phenyl vinyl silicone resin (refractive index of 1.5326, vinyl content of $5 \%$, volatility $<0.5 \%$ ), octaphenyl cyclotetrasiloxane (content of $99.5 \%$ ), tetramethyltetraphenylcyclotetrasiloxane (content of $99.5 \%$ ) and phenyltrimethoxysilane (content of 99.8\%) were purchased from Shaoguan YueYouYan Chemical Technology Co Ltd., China. Tetraethyl orthosilicate (TEOS, content of $99.8 \%$ ) was purchased from Xin'an Chemical Co Ltd., China. Dihydro-tetramethyl disiloxane (content of $99.7 \%$ ) was purchased from Guangzhou Gigantic Silicone Material Co Ltd., China. Dimethyl silicone oil (volatility $<0.5 \%$ ) and high-hydro silicone oil (hydrogen content of $1.58 \%$, volatility < $0.5 \%$ ) were purchased from Wacker Chemical Co Ltd., China. Hexamethyldisiloxane (content of 99.8\%) was purchased from Jiangxi Xinghuo Chemical Factory., China. All other reagents were chemically pure grade.

\subsection{Instruments}

Infrared Spectrometer (Model Nicolet 5700, by Thermo Electron). NMR (Model INOVA-300, by Varian). 
Abbe Refractometer (Model 2WA-J, by Shanghai Optical Instruments Factory, China). Gel Permeation Chromatography (Model 1260 Infinity, by Agilent). Hot and Cold Impact Tester (Model EET - 50L, by Dongguan Qingsheng Test Equipment Co Ltd., China). Reflow Tester (Model ZS-350, by Shenzhen Zhonghong Maituo Technology Co Ltd., China). Drying oven (Model DK - 136A, by Dongguan Haobang Industrial Equipment Co Ltd., China). Solid Crystal Microscope (Model XTJ-2600, by Shenzhen Osiwei Optical Instrument Co Ltd., China). Aging Tester (Model HP8000, by Hangzhou Hongpu Optoelectronics Technology Co Ltd., China).

\subsection{Synthesis of phenyl hydrogen polysiloxanes}

\subsubsection{Synthesis of phenyl hydrogen polysiloxanes with resin structures}

The following were added to a $2 \mathrm{~L}$ three-necked flask: $86.56 \mathrm{~g}$ of dihydro-tetramethyl disiloxane, $8.75 \mathrm{~g}$ of hexamethyldisiloxane, $514.8 \mathrm{~g}$ of phenyltrimethoxysilane and $400 \mathrm{~g}$ of methylcyclohexane. The mixture was stirred until uniform, and $10 \mathrm{~g}$ of $36.5 \% \mathrm{HCl}$ were added. Then $400 \mathrm{~g}$ of deionized water were slowly added to the flask via a constant pressure-dripping funnel, with time of addition being controlled within about 2 hours. After all the water had been added, the flask was heated to $70{ }^{\circ} \mathrm{C}$ and kept at that temperature for 3 hours, then cooled to room temperature. The organic phase was separated and washed with water until neutral. Then $20 \mathrm{~g}$ of sulfuric acid (content of $98 \%$ ) was added into the organic phase, and stirred for 4 hours at room temperature. Then $80 \mathrm{~g}$ of sodium carbonate was added to neutralize the sulfuric acid. The mixture was vacuum filtered and the solvent was removed to yield a colorless and transparent phenyl hydrogen polysiloxane, with hydrogen content of $0.30 \%$, refractive index of 1.5032 and viscosity of $300 \mathrm{mPa} \cdot \mathrm{s}$ (labeled as sample 1).

By altering the ratio of the reactants dihydrotetramethyl disiloxane, hexamethyldisiloxane and phenyltrimethoxysilane, a phenyl hydrogen polysiloxane of resin-structure with high viscosity was obtained. The hydrogen content of this phenyl hydrogen polysiloxane (labeled as sample 2) was $0.30 \%$, with its refractive index of 1.5235 and viscosity of $3500 \mathrm{mPa} \cdot \mathrm{s}$.

\subsubsection{Synthesis of phenyl hydrogen polysiloxanes with resin-resin structures}

The following were added to a $2 \mathrm{~L}$ three-necked flask: $33.5 \mathrm{~g}$ of dihydro-tetramethyl disiloxane, $99 \mathrm{~g}$ of phenyltrimethoxysilane and $100 \mathrm{~g}$ of methylcyclohexane. The mixture was stirred until uniform and then $5 \mathrm{~g}$ of p-toluenesulfonic acid were added. Then $100 \mathrm{~g}$ of deionized water were slowly added to the flask via a constant pressure-dripping funnel, with time of addition being controlled within about 2 hours. After all the water was added, the flask was heated to $70{ }^{\circ} \mathrm{C}$ and kept at that temperature for 3 hours, then cooled to room temperature. The organic phase was separated and washed with water until neutral, yielding the hydroxyl-containing MT-structure phenyl hydrogen silicone resin solution (Component A). In another 2L flask, $100 \mathrm{~g}$ of deionized water and $5 \mathrm{~g}$ of $\mathrm{p}$-toluenesulfonic acid were added and stirred until uniform. Then $88.6 \mathrm{~g}$ of TEOS was slowly added to the flask via a constant pressure-dripping funnel, with time of addition being controlled within about 1 hour. After all the TEOS was added, $100 \mathrm{~g}$ of methylcyclohexane and $34.5 \mathrm{~g}$ of hexamethyldisiloxane were added. The flask was heated to $70{ }^{\circ} \mathrm{C}$ and kept at that temperature for 3 hours, then cooled to room temperature. The organic phase was separated and washed with water until neutral, yielding the hydroxyl-containing MQ silicone resin solution (Component $\mathrm{B}$ ).

The Components A and B obtained above were mixed at the ratio of $1: 1$ and stirred until uniform, then $10 \mathrm{~g}$ of sulfuric acid (content of $98 \%$ ) were added and the mixture was stirred for 4 hours at room temperature. Then $40 \mathrm{~g}$ of sodium carbonate were added to neutralize the sulfuric acid. The mixture was vacuum filtered and the solvent was removed to yield a colorless and transparent phenyl hydrogen polysiloxane with a resin-resin structure. The hydrogen content of this polysiloxane was $0.29 \%$, with its refractive index of 1.5096 and viscosity of $1560 \mathrm{mPa} \cdot \mathrm{s}$ (labeled as sample 3).

\subsubsection{Synthesis of phenyl hydrogen polysiloxanes with resin-oil structures}

$8.75 \mathrm{~g}$ of hexamethyldisiloxane, $514.8 \mathrm{~g}$ of phenyltrimethoxysilane and $400 \mathrm{~g}$ of methylcyclohexane were added to a $2 \mathrm{~L}$ three-necked flask and stirred until uniform. Then $10 \mathrm{~g}$ of $36.5 \% \mathrm{HCl}$ were added. $400 \mathrm{~g}$ of deionized water were then slowly added to the flask via a constant pressure-dripping funnel, with time of addition being controlled within about 2 hours. After all the water had been added, the flask was heated to $70{ }^{\circ} \mathrm{C}$ and kept at that temperature for 3 hours, then cooled to room temperature. The organic phase was separated and washed with water until neutral. $20 \mathrm{~g}$ of sulfuric acid (content of $98 \%$ ) and $23 \mathrm{~g}$ of high hydrogen silicone oil were added to the organic phase and stirred for 4 hours at room temperature, then $80 \mathrm{~g}$ of sodium carbonate were added to neutralize the sulfuric acid. The mixture was vacuum filtered and the solvent was removed to yield a colorless transparent phenyl hydrogen polysiloxane with a resin-oil structure. The hydrogen content of the polysiloxane was $0.31 \%$, with its refractive index of 1.5105 and viscosity of $840 \mathrm{mPa} \cdot \mathrm{s}$ (labeled as sample 4).

\subsubsection{Synthesis of phenyl hydrogen polysiloxanes with oil structures}

$80 \mathrm{~g}$ of dimethyl silicone oil (viscosity of $5 \mathrm{mPa} \cdot \mathrm{s}$ ) and $320 \mathrm{~g}$ of octaphenyl cyclotetrasiloxane were added to a $2 \mathrm{~L}$ three-necked flask and stirred until uniform. Then $0.4 \mathrm{~g}$ of potassium hydroxide were added. The flask was heated to $150{ }^{\circ} \mathrm{C}$ and kept at that temperature for 8 hours, then cooled to room temperature. $20 \mathrm{~g}$ of sulfuric acid (content of $98 \%$ ), $400 \mathrm{~g}$ of methyl cyclohexane and $93.75 \mathrm{~g}$ of high hydrogen silicone oil were added and stirred for 4 hours at room temperature. Then $80 \mathrm{~g}$ of sodium carbonate were added to neutralize the sulfuric acid. The mixture was vacuum filtered and the solvent was removed to yield a colorless transparent phenyl hydrogen polysiloxane with an oil-structure. The hydrogen content of the polysiloxane was $0.29 \%$, with its refractive index of 1.5124 and viscosity oft $125 \mathrm{mPa} \cdot \mathrm{s}$ (labeled as sample 5). 


\subsection{Preparation of phenyl silicone rubbers}

Using phenyl vinyl silicone resin (refractive index of 1.5326, vinyl content of $5 \%$ ) as the base resin and controlling the ratio of $\mathrm{n}\left(\mathrm{Si}-\mathrm{CH}=\mathrm{CH}_{2}\right) / \mathrm{n}(\mathrm{Si}-\mathrm{H})$ at $1: 1.1$, the phenyl hydrogen polysiloxanes prepared above were added to the base resin respectively. The platinum catalyst was then added and the mixture was stirred until uniform. This mixture was then poured into a mold $(10 \mathrm{~cm}$ long $\times$ $4 \mathrm{~cm}$ wide $\times 4 \mathrm{~mm}$ thick), and vacuum deaerated until there were no residual air bubbles. Finally, it was placed in an oven and baked at $80^{\circ} \mathrm{C}$ for 1 hour and $150{ }^{\circ} \mathrm{C}$ for 3 hours sequentially, then cooled to room temperature.

The dumbbell silicone rubber samples were prepared according to GB/T528-2009, and their tensile strength was characterized.

\section{RESULTS AND DISCUSSION}

\subsection{FT-IR and ${ }^{1} \mathrm{H}-\mathrm{NMR}$ analysis of phenyl hydrogen polysiloxanes}

Fig. 1 shows the FT-IR spectra of the phenyl hydrogen polysiloxanes prepared in this study (samples 1-5).

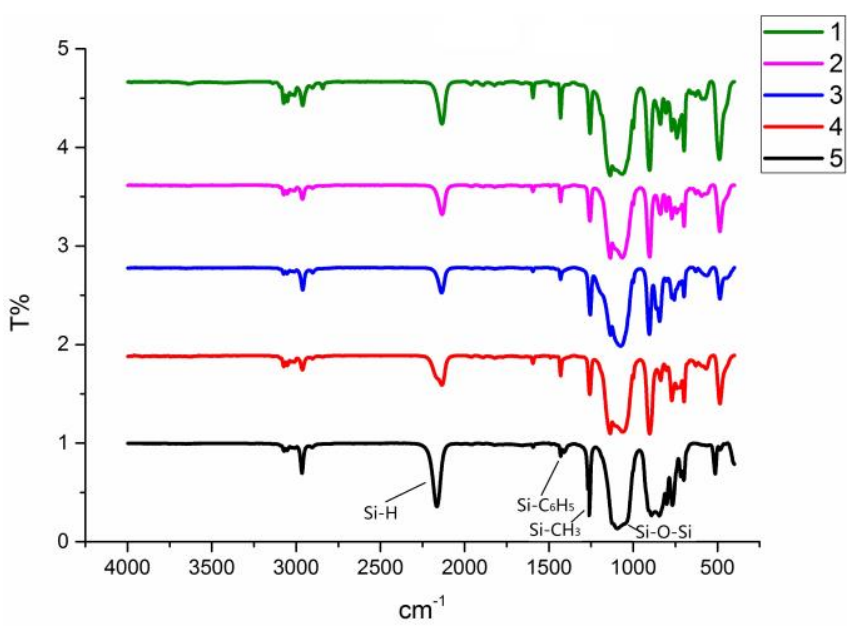

Fig. 1. FT-IR of phenyl hydrogen polysiloxane

As can be seen in Fig. 1, $1431 \mathrm{~cm}^{-1}$ was the characteristic peak of $\mathrm{Si}-\mathrm{Ph}$, while the peaks at $1255 \mathrm{~cm}^{-1}$, $1085 \mathrm{~cm}^{-1}$ and $2135 \mathrm{~cm}^{-1}$ were the characteristic peaks of $\mathrm{Si}-\mathrm{CH}_{3}, \mathrm{Si}-\mathrm{O}-\mathrm{Si}$, and $\mathrm{Si}-\mathrm{H}$, respectively. The absence of a characteristic $\mathrm{Si}-\mathrm{OH}$ peak at $3340 \mathrm{~cm}^{-1}$ due to silane hydrolyzates indicates that the products obtained in this study were phenyl hydrogen polysiloxanes.

Fig. 2 shows the ${ }^{1} \mathrm{H}-\mathrm{NMR}$ of the phenyl hydrogen polysiloxanes.
As can be seen in Fig. 2, chemical shift $\delta=7.0-7.5$ was the proton characteristic peaks of $\mathrm{Si}-\mathrm{Ph}$, while $\delta=4.5-5.0,0-1.0$ were the proton characteristic peaks of $\mathrm{Si}-\mathrm{H}$ and $\mathrm{Si}-\mathrm{CH}_{3}$, respectively.

\subsection{Structural analysis of phenyl hydrogen polysiloxanes}

The chemical structures of the polysiloxanes are shown in Fig. 3.

Where $\left[\mathrm{H} \quad\left(\mathrm{CH}_{3}\right)_{2} \mathrm{SiO}_{0.5}\right]$ and $\left[\left(\mathrm{CH}_{3}\right)_{3} \mathrm{SiO}_{0.5}\right]$ were $\mathrm{M}$-structure link, $\left[\left(\mathrm{CH}_{3}\right)_{2} \mathrm{SiO}\right]$ and $\left[\mathrm{H}\left(\mathrm{CH}_{3}\right) \mathrm{SiO}\right]$ were $\mathrm{D}$-structure link, while $\left[\mathrm{PhSiO}_{1.5}\right]$ and $\mathrm{SiO}_{2}$ were $\mathrm{T}$-structure link and $\mathrm{Q}$-structure link, respectively. Accordingly, samples 1 and 2 were of MT resin structure, sample 3 MTQ resin-resin structure, sample 4 MDT resinoil structure, and sample $5 \mathrm{MD}$ oil structure.

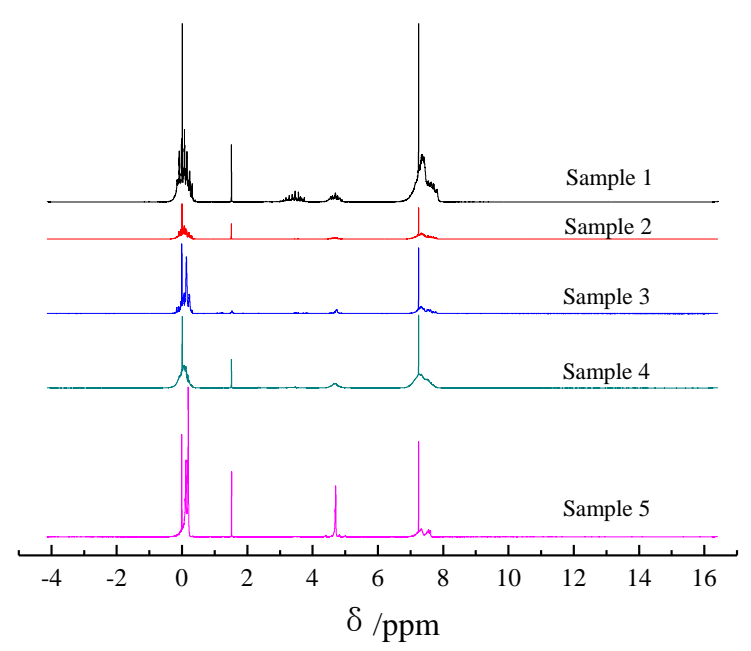

Fig. 2. ${ }^{1} \mathrm{H}-\mathrm{NMR}$ of the phenyl hydrogen polysiloxanes

\subsection{The molecular weight and distribution of the phenyl hydrogen polysiloxanes}

Table 1 shows the molecular weight and distribution of the five polysiloxanes prepared in this study. As can be seen from the Table 1, the resin-structure phenyl hydrogen polysiloxane has a lower molecular weight, whereas the oil-structure phenyl hydrogen polysiloxane has a higher molecular weight. It should be noted that while the viscosity of sample 2 is 10 times higher than sample 1 , their respective molecular weight is very close. Sample 3 has the lowest molecular weight, and sample 4 assumes a resin-oil structure and has a molecular weight higher than polysiloxanes of a resin structure.

Table 1. Molecular weight and distribution of the phenyl hydrogen polysiloxanes

\begin{tabular}{|c|c|c|c|c|c|c|c|}
\hline Sample & Mn & Mw & MP & Mz & $\mathrm{M}_{\mathrm{z}+1}$ & Polydispersity & $\%$ Area \\
\hline 1 & 1305 & 1553 & 987 & 1947 & 2500 & 1.1907 & 100.00 \\
\hline 2 & 1378 & 1746 & 1009 & 2400 & 3370 & 1.2666 & 100.00 \\
\hline 3 & 1146 & 1418 & 938 & 2574 & 6112 & 1.2376 & 100.00 \\
\hline 4 & 2676 & 6768 & 1502 & 15511 & 25303 & 2.5287 & 100.00 \\
\hline 5 & 16562 & 38926 & 26848 & 83192 & 142435 & 2.3503 & 100.00 \\
\hline
\end{tabular}




$$
\text { Sample1: }
$$

Fig. 3. Structural analysis of phenyl hydrogen polysiloxanes

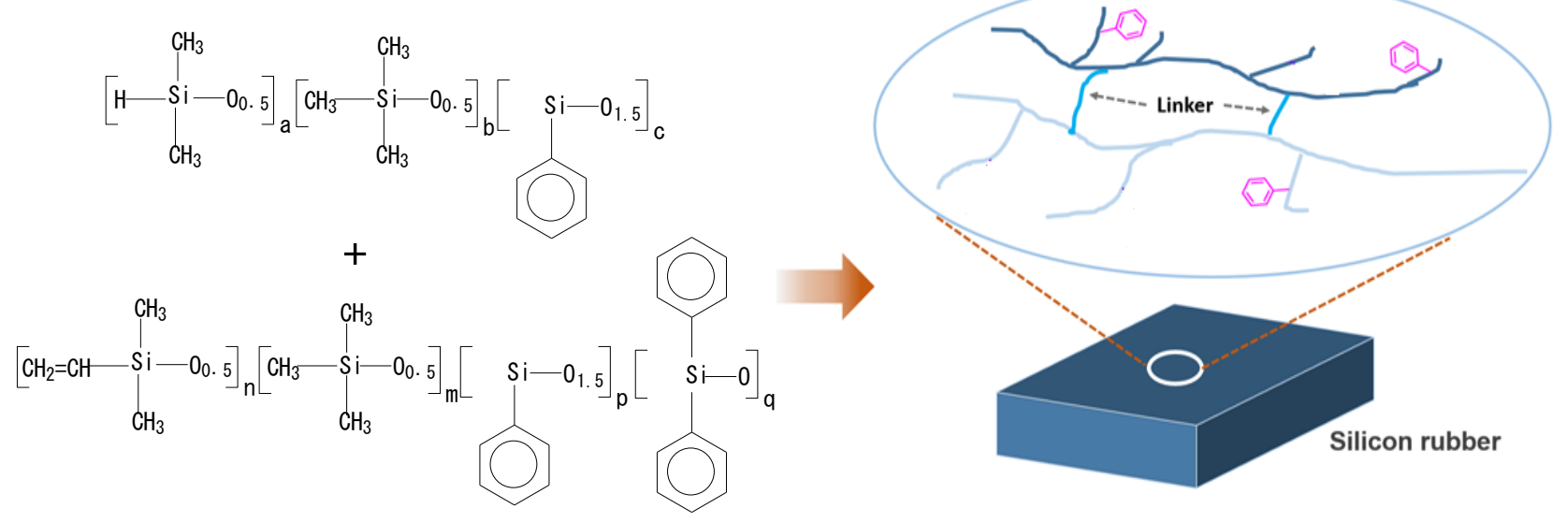

Fig. 4. Chemical structures of the staring materials and reaction scheme

\subsection{Heat resistance of phenyl hydrogen polysiloxanes}

Samples $1-5$ were heated at $300{ }^{\circ} \mathrm{C}$ for 24 hours, and the mass loss of the samples were measured to determine the thermal stability, the results were shown in Table 2.

As can be seen in Table 2, samples 1-5 had the similar mass loss after baking at $300{ }^{\circ} \mathrm{C}$ at the same time, because the introduction of phenyl to the molecular chains can effectively improve the heat resistance. The five samples had similar phenyl content, so they had the similar thermal stability.
Table 2. The thermal stability of phenyl hydrogen polysiloxanes

\begin{tabular}{|c|c|c|c|c|c|}
\hline Sample & 1 & 2 & 3 & 4 & 5 \\
\hline $\begin{array}{c}\text { Mass loss } \\
\left(300^{\circ} \mathrm{C} \times 1 \mathrm{~h}\right), \%\end{array}$ & 1.52 & 1.50 & 1.58 & 1.56 & 1.68 \\
\hline $\begin{array}{c}\text { Mass loss } \\
\left(300^{\circ} \mathrm{C} \times 5 \mathrm{~h}\right), \%\end{array}$ & 1.76 & 1.75 & 1.78 & 1.82 & 1.75 \\
\hline $\begin{array}{c}\text { Mass loss } \\
\left(300^{\circ} \mathrm{C} \times 10 \mathrm{~h}\right), \%\end{array}$ & 2.41 & 2.35 & 2.54 & 2.46 & 2.52 \\
\hline $\begin{array}{c}\text { Mass loss } \\
\left(300^{\circ} \mathrm{C} \times 24 \mathrm{~h}\right), \%\end{array}$ & 3.42 & 3.27 & 3.36 & 3.53 & 3.49 \\
\hline
\end{tabular}




\subsection{Effect of phenyl hydrogen polysiloxane on the hardness of silicone rubbers}

Chemical structures of the staring materials and reaction scheme was shown in Fig. 4.

The hardness, tensile strength, elongation and volume expansion coefficient of the rubbers prepared by different phenyl hydrogen polysiloxanes (crosslinking agents) were characterized, and the results are shown in Table 3.

Table 3. Mechanical properties of phenyl silicone rubbers

\begin{tabular}{|c|c|c|c|c|c|}
\hline $\begin{array}{l}\text { Cross- } \\
\text { linking } \\
\text { agent }\end{array}$ & Hardness & Hardness* & $\begin{array}{c}\text { Tensile } \\
\text { strength, } \\
\text { MPa }\end{array}$ & $\begin{array}{c}\text { Elongation, } \\
\%\end{array}$ & $\begin{array}{l}\text { Volume } \\
\text { expansion } \\
\text { coefficient } \\
\text { ppm }\end{array}$ \\
\hline Sample 1 & & & & & 262 \\
\hline Sample 2 & 55 & $\mathrm{I}$ & & & \\
\hline Sample 3 & D48 & $\mathrm{D}$ & & 32 & 265 \\
\hline Sample 4 & D45 & 8 & & 48 & 216 \\
\hline Sample 5 & $\mathrm{D} 25$ & D27 & 4.5 & 60 & 208 \\
\hline \multicolumn{6}{|c|}{$\begin{array}{l}\text { with the exception of this test which was performed using } \\
\text { amples cured sequentially at } 80^{\circ} \mathrm{C} \text { for } 1 \text { hour, } 150{ }^{\circ} \mathrm{C} \text { fo } \\
\text { hours and } 250^{\circ} \mathrm{C} \text { for } 24 \text { hours, all other tests used sample } \\
\text { ured sequentially at } 80{ }^{\circ} \mathrm{C} \text { for } 1 \text { hour and } 150{ }^{\circ} \mathrm{C} \text { for } 3 \text { hours }\end{array}$} \\
\hline
\end{tabular}

As can be seen from Table 3, for samples cured sequentially at $80{ }^{\circ} \mathrm{C}$ for 1 hour and $150{ }^{\circ} \mathrm{C}$ for 3 hours, the hardness of the silicone rubbers differed considerably even though they had the same proportion of phenyl hydrogen polysiloxanes. Two structural aspects of a phenyl hydrogen polysiloxane contribute to the hardness of the silicone rubbers formed from the polysiloxane. The first is the crosslinking matrices formed from the hydrogen and vinyl reactions; more hydrogens in the polysiloxane lead to a higher level of crosslinking, and thus a greater hardness. The second is the resin structures contained in the polysiloxane, which form a high degree of crosslinking network, reinforcing and enhancing the hardness. The reinforcing impact of the resin structures on the hardness is directly related to the crosslinking density in the resin structures; the greater the crosslinking density, the greater the enhancement to the hardness. Therefore, the silicone rubber cured by sample 5 exhibited the lowest hardness, as its hardness derived only from the hydrogen and vinyl reaction. All other silicone rubbers exhibited a higher hardness than that of sample 5, since they contained resin structures. Likewise, sample 2 generated a greater crosslinking density than sample 1, and thus a higher hardness. Similarly, there is a larger number of resin-structures in sample 3 than sample 1, thus a higher hardness.

The hardness of the silicone rubbers improved upon further curing at $250{ }^{\circ} \mathrm{C}$ for 24 hours after curing sequentially at $80{ }^{\circ} \mathrm{C}$ for 1 hour and $150{ }^{\circ} \mathrm{C}$ for 3 hours. The hardness of the rubbers increased by 11-12D for Samples 1, 2 and 3, and 2-3D for samples 4 and 5. The phenyl groups in the polysiloxanes encounter significant steric hindrance, blocking the reaction of the vinyl and hydrogen groups. This steric hindrance is further strengthened in polysiloxanes with resin structures which act to increase the hindrance effect. Under the curing condition of sequential $80^{\circ} \mathrm{C}$ for 1 hour and $150{ }^{\circ} \mathrm{C}$ for 3 hours, the vinyl and hydrogen groups could not react fully. Further curing at $250^{\circ} \mathrm{C}$ for 24 hours would enable the remaining vinyl and hydrogen groups to react, resulting in an increased hardness. The phenyl groups in phenyl hydrogen polysiloxanes with oil structure or oil-resin structure were more evenly distributed, encountering less steric hindrance. Therefore, the reaction of the vinyl and hydrogen groups involving these polysiloxanes was almost completed upon sequential curing at $80{ }^{\circ} \mathrm{C}$ for 1 hour and $150^{\circ} \mathrm{C}$ for 3 hours, and further curing at $250^{\circ} \mathrm{C}$ for 24 hours generated insignificant increase in hardness.

Hardness increasing of the packaging silicone rubber encountered in LED lighting applications is a fatal problem for LED lamps, as it will lead to decreased tensile strength and elongation, and eventually cracking of the silicone rubber.

\subsection{Effect of phenyl hydrogen polysiloxane on the mechanical properties of silicone rubbers}

As can be seen in Table 3 , the rubbers cured by samples 1, 2 and 3 had better tensile strength, lower elongation and higher volume expansion coefficient, while the rubber cured by sample 5 had the lowest tensile strength, highest elongation and lowest volume expansion coefficient. The rubber cured by sample 4 had moderate tensile strength, elongation and volume expansion coefficient.

The performance of packaging silicone rubber is closely correlated with its tensile strength, elongation and volume expansion coefficient. Low tensile strength and elongation indicate a lack of elasticity and toughness, rendering the rubber to be easily damaged by external factors. Rubbers with a high volume expansion coefficient undergo high volume change during hot and cold impact cycles, making them crack easily and the gold lines contained wherein ripped.

Samples 1, 2 and 3 contained only resin structure which imparted a positive reinforcement effect. While the resin structure enhanced the cohesion and tensile strength of the silicone rubbers, it also reduced their elongation and increased their volume expansion coefficient. Sample 5 contained only oil structure, and the cured silicone rubber made from it exhibited lower cohesion, higher tensile strength and lower volume expansion coefficient, rendering effect of temperature on the volume change in this rubber to be minimal. In contrast, sample 4 combined the advantages of both the resin and oil structures.

\subsection{Effect of phenyl hydrogen polysiloxane on yellowing of silicone rubber}

While phenyl packaging silicone rubbers exhibit some desirable properties such as high refractive index, high heat resistance and hardness, they are also prone to yellowing during the aging process, resulting in light attenuation. To characterize the behavior of yellowing, phenyl vinyl silicone resin, phenyl hydrogen polysiloxanes and the cured silicone rubbers were baked at $280{ }^{\circ} \mathrm{C}$ for 24 hours, and the color change was observed. The results are shown in Table 4. As can be seen in Table 4, five phenyl hydrogen polysiloxane samples did not undergo color change upon curing at $300{ }^{\circ} \mathrm{C}$ for 24 hours, but the color of the cured rubbers turned yellow, particularly seriously for 
the rubbers made from samples 1,2 and 3. The phenyl vinyl silicone resin turned brown, which indicated that yellowing of the silicone rubbers was most probably due to the residual vinyl and was not related to the phenyl groups.

Table 4. Color change upon heat treatment at $280{ }^{\circ} \mathrm{C}$ for 24 hours

\begin{tabular}{|c|c|c|}
\hline Sample & Polysiloxanes & $\begin{array}{c}\text { Silicone rubbers } \\
\text { made from } \\
\text { polysiloxanes }\end{array}$ \\
\hline 1 & colorless $\rightarrow$ colorless & colorless $\rightarrow$ yellow \\
\hline 2 & colorless $\rightarrow$ colorless & colorless $\rightarrow$ yellow \\
\hline 3 & colorless $\rightarrow$ colorless & colorless $\rightarrow$ yellow \\
\hline 4 & colorless $\rightarrow$ colorless & colorless $\rightarrow$ yellowish \\
\hline 5 & colorless $\rightarrow$ colorless & colorless $\rightarrow$ yellowish \\
\hline $\begin{array}{c}\text { Phenyl vinyl } \\
\text { silicon resin }\end{array}$ & colorless $\rightarrow$ brown & \\
\hline
\end{tabular}

Under the condition of curing sequentially at $80{ }^{\circ} \mathrm{C}$ for 1 hour and $150{ }^{\circ} \mathrm{C}$ for 3 hours, the vinyl was unable to react completely, with the residual vinyl being oxidized and turning yellow at high temperatures. More residual vinyl there is in the rubbers, the more severe the yellowing. For phenyl hydrogen polysiloxanes with an oil structure, the vinyl groups can react more completely with the phenyl groups as the latter was more evenly distributed. As a result, less unreacted vinyl remained and less serious yellowing occured.

\subsection{Effect of phenyl hydrogen polysiloxane on sulfuration resistance of silicone rubbers}

The main chain of methyl silicone rubbers has a helical structure, rendering great freedom of methyl activities. Methyl silicone rubbers have good permeability, and gas can freely permeate. Introduction of phenyl groups in the structure increases the electron density, which reduces the freedom of methyl activities and permeability, thus improving the ability of sulfuration resistance.

The LED packaging silicone rubbers cured by different phenyl hydrogen polysiloxanes were prepared, and packaged on 3528 chips. $10 \mathrm{~g}$ of sublimed sulfur were placed in a $1000 \mathrm{~mL}$ beaker, followed by placement of 10 lamps in the beaker. The beaker was sealed and placed into an oven at $100{ }^{\circ} \mathrm{C}$ and heated for 24 hours. The optical power of the lamps before and after sulfuration was measured to characterize the sulfuration resistance of the rubbers. The results of the photoelectric tests of the lamps before and after sulfuration are shown in Table 5 .

Table 5. Optoelectronic test of lamps before and after sulfuration

\begin{tabular}{|c|c|c|c|c|}
\hline $\begin{array}{c}\text { Selection of } \\
\text { crosslinking } \\
\text { agent }\end{array}$ & $\begin{array}{c}\text { Number } \\
\text { of } \\
\text { lamps }\end{array}$ & $\begin{array}{c}\text { Average } \\
\text { optical } \\
\text { power } \\
\text { before } \\
\text { sulfuration, } \\
\text { W } \times 10^{-2}\end{array}$ & $\begin{array}{c}\text { Average } \\
\text { optical } \\
\text { power after } \\
\text { sulfuration, } \\
\text { W } \times 10^{-2}\end{array}$ & $\begin{array}{c}\text { Average } \\
\text { flux } \\
\text { maintain } \\
\text { rate after } \\
\text { sulfuration, } \\
\%\end{array}$ \\
\hline 1 & 10 & 2.188 & 1.775 & 81.12 \\
\hline 2 & 10 & 2.173 & 1.893 & 87.11 \\
\hline 3 & 10 & 2.322 & 1.989 & 85.66 \\
\hline 4 & 10 & 2.233 & 1.859 & 83.25 \\
\hline 5 & 10 & 2.236 & 1.772 & 79.25 \\
\hline
\end{tabular}

As can be seen in Table 5, the silicone rubbers cured by samples 2 and 3 exhibited superior anti-sulfuration ability, the luminous flux was maintained at more than $85 \%$. The silicone rubbers cured by samples 4 and 1 had a similar anti-sulfuration ability and the luminous flux was maintained at $80 \sim 83 \%$. The silicone rubber cured by sample 5 showed the worst anti-sulfuration behavior, with the luminous flux at $77 \sim 79 \%$ after sulfuration. The different anti-sulfuration behavior observed can probably be explained by the structural difference in the silicone rubbers used. Samples 1, 2, 3 and 4 contained resin structure and had higher crosslinking density, which reduced gas permeability and imparted superior antisulfuration properties.

\subsection{Effect of phenyl hydrogen polysiloxane on hot and cold impact cycles}

The LED packaging silicone rubbers cured by phenyl hydrogen polysiloxanes was prepared and packaged on 3528 chips. 10 pieces of the chips were used to perform the hot and cold impact cycle test. The test conditions were as follows: $-40^{\circ} \mathrm{C} \times 15 \mathrm{~min} \sim 120^{\circ} \mathrm{C} \times 15 \mathrm{~min}$ ( 1 cycle). The chips were taken out every 10 cycles and tested to observe whether the lamps could be lit by using a constant current power supply. The test results are shown in Table 6.

Table 6. Hot and cold impact test of packaged lamps

\begin{tabular}{|c|c|c|c|c|c|}
\hline $\begin{array}{c}\text { Selection of } \\
\text { crosslinking } \\
\text { agent }\end{array}$ & 1 & 2 & 3 & 4 & 5 \\
\hline Cycle times & 220 & 210 & 180 & 450 & 500 \\
\hline
\end{tabular}

As can be seen in Table 6, lamps packaged with the silicone rubbers cured by samples 4 and 5 exhibited much higher cycle times than those by samples 1,2 and 3 . Samples 1, 2 and 3 contained resin structure, and their hardness increased significantly under hot and cold impact cycles. Their volume expansion coefficient was also greater (see Table 3), which caused the silicone rubbers to undergo significant expansion and contraction repeatedly. This alternate expansion-contraction occurrence induced a large volume change and resulted in tear-off of the gold lines and/or cracking of the rubbers. The hardness of silicone rubbers cured by samples 4 and 5 remained basically unchanged during the hot-cold cycles, and their volume expansion coefficient was also smaller, together making the effect of hot and cold impact cycles on the performance of these rubbers much less significant.

\section{CONCLUSIONS}

Phenyl hydrogen polysiloxane have effects on the hardness, mechanical properties, yellowing, anti-sulfuration, hot and cold impact. Studying the effects of phenyl hydrogen polysiloxane structures on the performance of phenyl silicone rubbers has important significance for the design, synthesis and application of LED packaging silicone rubbers. The results are helpful to solve the problems (e.g. change in hardness, yellowing, gas permeation and sulfuration, and high volume expansion coefficient) encountered in application of silicone rubbers 
in LED packaging, and extend LED service lives, which make the application more convenient and reliable.

\section{Acknowledgments}

This study was supported by Fund of Applied Science and Technology Research in Guangdong Province, China (2017A070701024, 2017A070702020), Special Fund Project for Technology Development of Guangdong Province, China (2016B070701023), Fund of Guangdong Academy of Sciences, China (2018GDASCX-0942).

\section{REFERENCES}

1. Joo, J., Lee, C.Y. High Frequency Electromagnetic Interference Shielding Response of Mixtures and Multilayer Films Based on Conducting Polymers Journal of Applied Physics 88 (1) 2000: pp. 513-518. https://doi.org/10.1063/1.373688

2. Grassie, N., Macfarlane, I.G., Francey, K.F. The Thermal Degradation of Polysiloxanes European Polymer Journal 15 1979: pp. 415 -422. https://doi.org/10.1016/0014-3057(78)90084-8

3. Kang, D.W., Yeo, H.G., Lee. K.S. Preparation and Characterisation of Liquid Silicone Rubber Nanocomposite Containing Ultrafine Magnesium Ferrite Powder Journal of Inorganic and Organometallic Polymers 14 (1) 2004: pp. $73-84$.

4. Birkefeld, A.B., Eckert, H., Pfleiderer. B. A Study of Aging Silicone Breast Implants Using ${ }^{29} \mathrm{Si}, \quad{ }^{1} \mathrm{H}$
Relaxation and DSC Measurements Biomaterials $25(18)$ 2004: pp. $4405-4413$.

https://doi.org/10.1016/j.biomaterials.2003.10.093

5. Zhang, T. Effect of Magnetic Field of Light on Refractive Index Chinese Physics 13 (8) 2004: pp. 1358-1364.

6. Zhang, T. A Possible Mechanism of Current in Medium under Electromagnetic Wave Chinese Physics $15(8)$ 2006: pp. $1752-1758$. https://doi.org/10.1088/1009-1963/15/8/020

7. Leonidas, C., Rafiqul, G. New Group Contribution Method for Estimating Properties of Pure Compounds AIChE Journal 40 (10) 1994: pp. 1697-1710. https://doi.org/10.1002/aic.690401011

8. David, W.B., Karl, K. Separation of Gases by Means of Permeable Membranes Industrial \& Engineering Chemistry Research 44 (6) 1952: pp. 1465-1474. https://doi.org/10.1021/ie50510a071

9. Kojima, A., Hoshii, S., Muto, T. Characteristics of Polysilazane Compound and its Application as Coating for Carbon Material Journal of Materials Science Letters 21 (10) 2002: pp. 757-760. https://doi.org/10.1023/A:1015709818240

10. Gnthner, M., Kraus, T., Dierdorf, A. Advanced Coatings on the Basis of $\mathrm{Si}(\mathrm{C}) \mathrm{N}$ Precursors for Protection of Steel Against Oxidation Journal of the European Ceramic Society 29 (10) 2009: pp. 2061-2068. https://doi.org/10.1016/j.jeurceramsoc.2008.11.013 